\title{
Facial Aesthetics in Adults and Its Relationship to "I deal" Ratios and Angles - A Photogrammetric Study In Hyderabad Population
}

\author{
Hari Prasad Rao G ${ }^{1}$, Padma Priya ${ }^{2}$, Ravindra Naik ${ }^{3}$
}

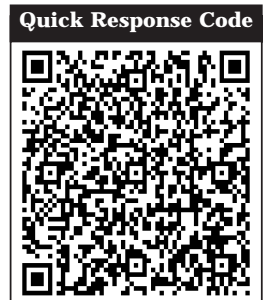

doi : 10.5866/2015.7.10173

${ }^{1}$ Professor and $\mathrm{HOD}$

2\&3P.G.

Dept. of Orthodontics,

Govt. Dental College and Hospital

Hyderabad.

\section{Article Info:}

Received: J uly 14, 2015

Review Completed: August 12, 2015

Accepted: September 11, 2015

Available Online: J uly, 2015 (www.nacd.in)

(C) NAD, 2015 - All rights reserved

\section{E mail for correspondence:}

harigutti@gmail.com

\begin{abstract}
:
STUDY BACKGROUND: Facial aesthetics is receiving ever increasing attention in orthodontics. Many guidelines, norms, and ideal ratios and angles for attractive faces have been proposed in the literature. The use of these population norms can be logically enhanced by evaluating normal ranges of variability in every aspect of craniofacial form, depending on age, sex, and ethnic variability. The purpose of this study was to establish facial soft tissue norms for Hyderabad young adults.

METHODS: Anthropometric measurements of thefacial soft tissue were taken from 80 Hyderabad young adults. 15 ratios and 15 angles were calculated on each standardized photograph, taken in a natural head position, and their deviation from the ideal targets in the literature was determined.
\end{abstract}

RESULTS: Ten Ratios and two Angles showed significant sexual dimorphism .Eight of the 15 investigated ratios on frontal photographs of males, 11 of the 15 investigated ratios on frontal photographs of females showed statistically significant differences.F ourteen of the 15 investigated angles on lateral photographs showed significant differences between Hyderabad population and Caucasians.

CONCLUSION: The results of the study support the fact that norms and standards of one racial group could not be used without modification for other racial group and each different racial group would have to be treated according to its individual characteristics.

Key words: Photogrammetry; I ntution; Caucasians; Profilometric analysis; Sexual dimorphism.

\section{Introduction:}

Facial aesthetics is receiving ever increasing attention in orthodontics. Wa hl wrote, "Now it appears that facial aesthetics is again in the for efront as we realize why patients come to us in the first place". ${ }^{1}$ The normal human face is possibly the most beautifully perfect structure in all of the animal kingdom. ${ }^{2}$ The human face holds an absorbing and consuming interest for people the world over. Our faces unquestionably influence and mould our character, personality, and behaviour. Orthodontics is a combination of both art and 
science, and facial aesthetics is the reflection of the orthodontist's artistic intuition. Facial features have been evaluated with anthropometric, photogrammetric and cephalometric measurements..$^{3-7}$. Because the standards of beauty could vary considerably among persons as well as racial groups, it is essential for a clinician to devel op the concept of normal for a particular racial group. ${ }^{8}$, 9 Hence, the present study was undertaken to evaluate the photogrammetric norms in cranio-facial region, which will prove useful to orthodontists, maxillo-facial and plastic surgeons.

\section{Aims and objectives:}

In the present cross-sectional study, the aim was to:

1. To analyze various facial measurements in local Hyderabad population and establish photogrammetric norms.

2. To compare male and female norms of Hyderabad population.

3. To compare these norms with the norms of other ethnic groups.

4. To derive clinical implications which will be useful to Orthodontists, Oral and Maxillofacial and Plastic Surgeons.

\section{Materials \& Methods:}

The material consisted frontal and lateral photographs of 80 young adults (40 females, mean age 21.15 years; 40 males, mean age 21.55 years) of native Hyderabad population. The inclusion criteria were age between 18 and 25 years, Acceptable, pleasing profiles with Class I molar relationship on both the sides, with normal over jet and overbite, not wearing glasses. No dental or facial trauma, and no congenital defects. All the subjects had completed their active physical growth. No history of previous Orthodontic or Prosthodontic treatment or facial surgery. The sample included both male and female sexes in equal numbers to evaluate the significant morphological differences between them.

\section{Photographic set up:}

The method described by Riverio et al (2003) for the photographic set up and record taking was used..$^{10}$ The photographic setup consisted of a tripod that held a 35-mm camera with a $100-\mathrm{mm}$ macro lens and a primary flash. For illumination during photography, umbrella flashes were used. The 100$\mathrm{mm}$ macro lens was chosen to avoid facial deformations. The stability of the elements and the easy adjustment of the tripod height allowed us to keep the optic axis of the lens horizontal during the recording. Levelling devices at the base of the tripod and on the camera controlled its correct horizontal position. The camera was used in its manual position, the shutter speed was 1/125 second, and the opening of the diaphragm was $f / 11$. Camera to subject distance was standardized at 1.5 meters.

\section{Patient Positioning:}

A portable cephal ostat was specifically confected for the present study and consisted of a metal structure with an acrylic part to which the ear rods were attached. These structures were adjustable in the vertical direction for the correct adaptation to the patient's ears. The photographs were obtained in both frontal and profileviews. For the profileview, the subject was instructed to sit on an adjustable stool in front of the mirror which was placed approximately $110 \mathrm{~cm}$ from subject, with his/her feet a short distance apart, back straight and looking into his/ her eyes in themirror. Once the patient remained stable in the NHP, the ear rods were inserted with light skin contact. ${ }^{11} \mathrm{~F}$ or frontal photographs, the subject was asked to look directly at the camera in a relaxed manner. The camera should be about at the height of the middle of the face and in portrait format. ${ }^{12}$

The photographic analysis was carried out on the computer using AutoCAD 2012 software. On the frontal photographs, 12 landmarks and, on the lateral photographs, 10 landmarks were selected. ${ }^{13}$ Fifteen ratios (indices) that express sizeindependent facial proportions are calculated on frontal photographs. Fifteen angular measurements were calculated on the lateral photographs and 15 Proportional Indices were Investigated on frontal photographs (Table 1 and 2).

Ratio 1: Tr-N/N-St, ratio between height of forehead and upper face.

Ratio 2: Tr-N/Sn-Me, ratio between height of forehead and lower face. 
Ratio 3: N-St/Sn-Me, ratio between upper face and lower face height.

Ratio 4: Sn-St/Sn-Me, ratio between upper lip and lower face height.

Ratio 5: St-Me/Sn-Me, ratio between lower lip and lower face height.

Ratio 6: Sn-St/St-Me, ratio between upper and lower lip length.

Ratio 7: Ls-St/St-Li, Vermilion Height I ndex.

Ratio 8: AIR-AIL/ChR-ChL, Nose-Mouth width Index.

Ratio 9: ChR-ChL/XR-XL, Mouth- face width Index.

Ratio 10: AIR-AIL/ N-Sn, Nasal Index.

Ratio 11: Sn-St/ChR-ChL, U pper lip length-M outh width Index.

Ratio 12: Sn-Me/ChR-ChL, Lower face heightMouth width Index.

Ratio 13: Sn-Me/XR-XL, Lower face I ndex.

Ratio 14: N-St/XR-XL, U pper face I ndex.

Ratio 15: N-Me/XR-XL, Facial Index.

Investigated 15 Angles on Lateral photographs

Angle 1: Lsp-G-Pog; Upper lip projection.

Angle 2: Lip-G-Pog; Lower lip projection.

Angle 3: Lsp-N-Pog; Maxillofacial angle.

Angle 4: G-N-Prn; Nasofrontal angle.

Angle 5: Prn-N-Sn;Columella length angle.

Angle 6: Prn-S-Pog; Nasal prominence angle.

Angle 7: N-Prn-Pog.

Angle 8: G-Sn-Pog; Angle of facial convexity.

Angle 9: N-Sn-Pog.

Angle 10: N-Trg-Prn; Nasal angle.

Angle 11: N-Trg-Sn; Angle of medium facial third.

Angle 12: N-Trg-Pog; Total vertical angle.

Angle 13: N-Trg-Ls.

Angle 14: Sn-Trg-Me; Angle of inferior facial third.

Angle 15: Ls-Trg-Pog: Mandibular angle.

\section{Statistical analysis:}

The measurements were statistically analyzed by calculating their mean and standard deviation for both groups. A comparison was also made between males and females with the help of Student's unpaired ' $\mathrm{t}$ ' test, then the means of Hyderabad population were compared with means of Caucasian population with the help of OneSample t' test.

\section{Results:}

Ten Ratios and 2 Angles showed significant sexual dimorphism (Tables 3 and 4). Tr-N/N-St, ratio between height of forehead and upper face $(P=0.00)$. Tr-N/Sn-Me, ratio between height of forehead and lower face $(P=0.00)$. N-St/Sn-Me, ratio between upper face and lower face height $(P=0.00)$. Ls-St/St-Li, Vermilion Height I ndex $(P=0.05)$. AIRAI L/ N-Sn, Nasal Index ( $P=0.01)$. Sn-St/ChR-ChL, Upper lip length-M outh width Index $(P=0.00)$. SnMe/ChR-ChL, Lower face height- Mouth width Index $(P=0.00)$. Sn-Me/XR-XL, Lower face Index $(P=0.00)$. N-St/XR-XL, Upper face Index $(P=0.04)$. N-Me/XR-XL, Facial Index ( $P=0.00)$. Pn-S-Pog; Nasal prominence angle $(P=0.02)$, Ls-Trg-Pog: Mandibular angle $(P=0.03)$.

Eight of the 15 investigated ratios on frontal photographs of males showed significant statistical differences (Table 5). They are Ratio 6: Sn-St/StMe, ratio between upper and lower lip length $(P=0.00)$, Ratio7: Ls-St/St-Li, Vermilion Height Index ( $P=0.00)$, Ratio 8: Al R-AI L/ChR-ChL, N oseMouth widthIndex $(P=0.00)$, Ratio 9: ChR-ChL/ XR-XL, Mouth- face width Index $(P=0.00)$, Ratio 10: AIR-AIL/ N-Sn, Nasal Index $(P=0.00)$, Ratio 13: Sn-Me/XR-XL, Lower face Index $(P=0.00)$, Ratio 14: N-St/XR-XL, U pper face I ndex $(P=0.00)$, Ratio 15: N-Me/XR-XL, F acial Index $(P=0.00)$.

Eleven of the 15 investigated ratios on frontal photographs of females showed significant statistical differences (Table 6). They were:

Ratio 1: Tr-N/N-St, ratio between height of forehead and upper face ( $P=0.00)$,

Ratio 2: $\mathbf{T r}-\mathbf{N} / \mathbf{S n}-\mathbf{M e}$, ratio between height of forehead and lower face $(P=0.00)$, 
Ratio 3: N-St/Sn-Me, ratio between upper face and lower face height $(P=0.00)$, are

Ratio 6: Sn-St/St-Me, ratio between upper and lower lip length $(P=0.00)$,

Ratio 7: Ls-St/St-Li, Vermilion Height Index $(P=0.00)$,

Ratio 8: AIR-AIL/ChR-ChL, Nose-Mouth widthl ndex $(P=0.00)$,

Ratio 9: ChR-ChL/XR-XL, Mouth- face width Index. $(P=0.00)$,

Ratio 10: AIR-AIL/ N-Sn, Nasal I ndex ( $P=0.00)$,

Ratio 13: Sn-Me/XR-XL, Lower face Index $(P=0.00)$,

Ratio 14: N-St/XR-XL, U pper face I ndex $(P=0.00)$,

Ratio15: N-Me/XR-XL, Facial I ndex ( $\mathrm{P}=0.00)$.

Fourteen of the 15 investigated angles on lateral photographs showed significant differences between Hyderabad population and Caucasians (Tables7 and 8).

Angle1: Lsp-G-Pog; U pper lip projection ( $P=0.04)$,

Angle2: Lip-G-Pog; Lower lip rojection ( $P=0.02)$,

Angle 3: Lsp-N-Pog; Maxillofacial angle $(P=0.00)$,

Angle4:G-N-Prn;Nasofrontal angle $(P=0.00)$,

Angle 5: Prn-N-Sn;Columella length angle $(P=0.00)$,

Angle 6: Prn-S-Pog; Nasal prominence angle $(P=0.00)$,

Angle 8: G-Sn-Pog; Angle of facial convexity $(P=0.00)$,

Angle 9: N-Sn-Pog $(P=0.00)$,

Angle 10: N-Trg-Prn; Nasal angle ( $P=0.00)$,

Angle 11: N-Trg-Sn; Angle of medium facial third $(P=0.00)$,

Angle 12: N-Trg-Pog; Total vertical angle $(P=0.00)$,

Angle 13: N-Trg-Ls $(P=0.00)$,

Angle 14: Sn-Trg-Me; Angle of inferior facial third $(P=0.00)$,

Angle 15: Ls-Trg-Pog: Mandibular angle $(P=0.00)$

\section{Discussion:}

\section{Frontal Photographic Analysis:}

The first step in analysing facial proportions is to examine the face in frontal view. ${ }^{14}$ Prior to the advent of cephalometric radiography, dentists and orthodontists often used anthropometric measurements (i.e., measurements made directly during the clinical examination) to help establish facial proportions. Although for orthodontists, this method was largely replaced by cephalometric analysis for many years, the recent emphasis on soft tissue proportions has brought soft tissue evaluation back into prominence. Differences in facial types and body types obviously must be taken into account when facial proportions are assessed, and variations from the average ratios can be compatible with good facial aesthetics.

In orthodontics, Ricketts was the first to claim that the analysis of a physically beautiful face should be approached mathematically. ${ }^{2}$ He reported that three values in attractive faces were approximately even. They were the forehead to the eye, the eye to the mouth, and the nose to the chin. The study demonstrated that Hyderabad males (Tr-N/N-St, (Figure 1, colour plate I) males $=1.02 \pm 0.09$, females $=1.13 \pm 0.07, P=0.00$; Tr-N/Sn-Me, (F igure 2, colour plate I) males $=1.01 \pm 0.1$, females $=1$ $.22 \pm 0.14, P=0.00 ; \mathbf{N}-S t / S n-M e$, (Figure 3, colour plate I) males $=0.99 \pm 0.06$, females $=1.04 \pm 0.07$, $\mathrm{P}=0.00$.) displayed the same proportions as that of Caucasians but females showed statistically significant differences (Females; Tr-N/N-St, $\mathrm{P}=0.00$; Tr-N/Sn-Me, $\mathrm{P}=0.00, \mathrm{~N}-\mathrm{St} / \mathrm{Sn}-\mathrm{Me}, \mathrm{P}=0.00$ ). The Hyderabad females displayed a significant increased ratio over the males, possibly dueto increased height of forehead, and greater distance from nasion to stomion. As the top of the forehead is marked by the variable position of the frontal hairline, which differs greatly between males and females, a large forehead does not necessarily mean that they have a large head overall, sometimes it means that hairline of females is further up their head. The ratio between upper lip height and lower face height (SnSt/Sn-Me, (Figure 4, colour plate I) males= $0.31 \pm 0.02$, females $=0.31 \pm 0.02$ ), lower lip height and lower face height (St-Me/ Sn-Me, (F igure 5, colour 

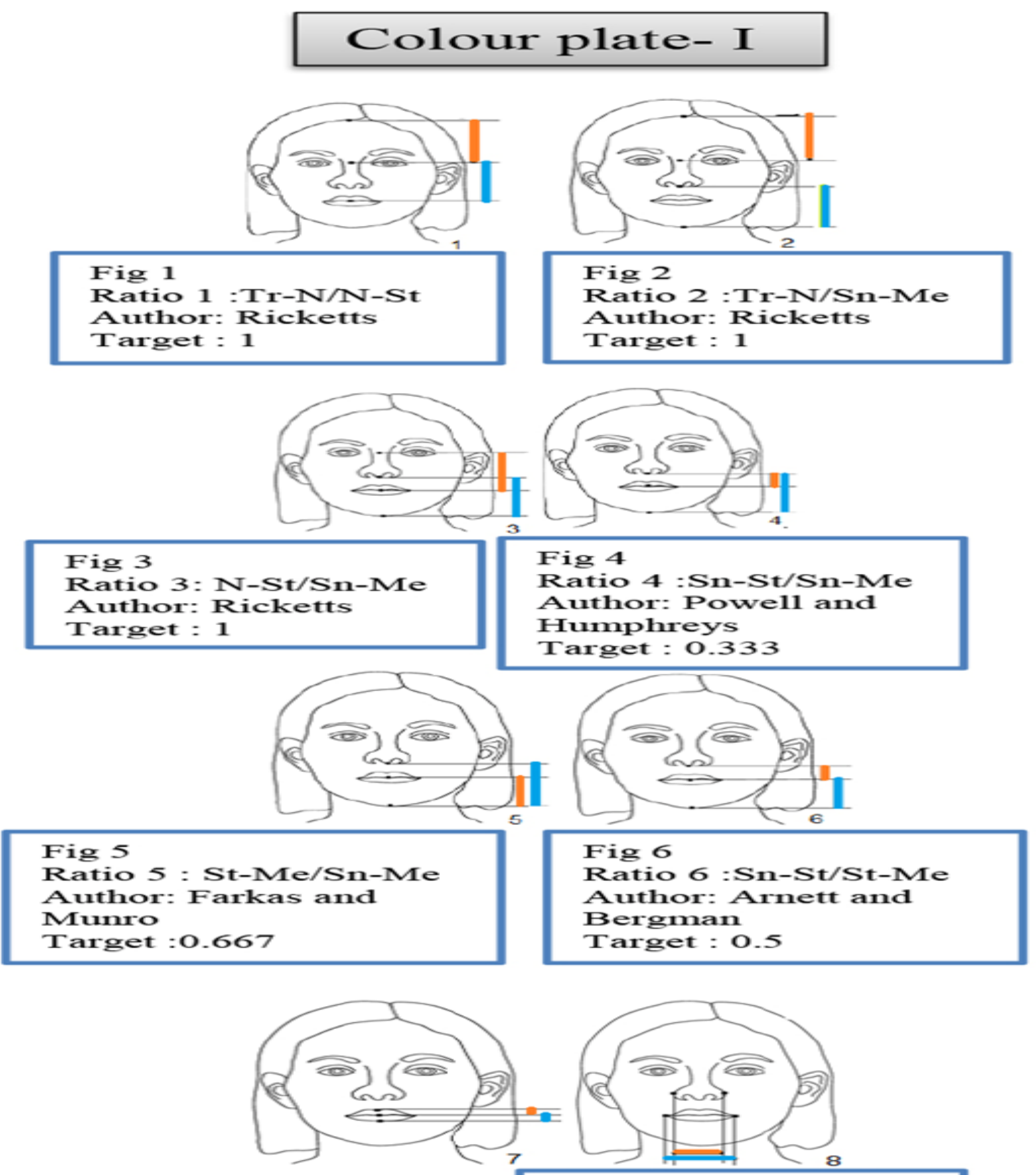

Fig 7

Ratio $7=I$ S-St/St-Ii

Author: Farkas et al

Target $=0.36$
Fig 8

Ratio 8 : AIR-

AIL/ChR-ChI

Author: Koury and

Epker

Target $=0.625$ 


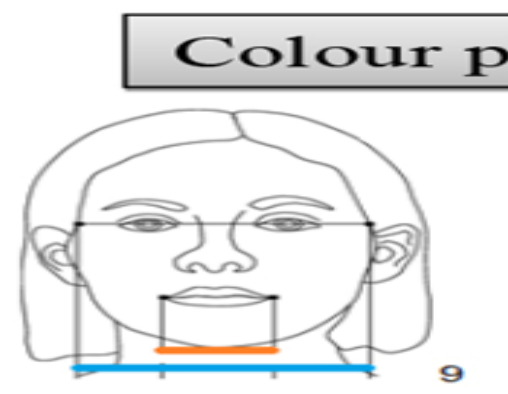

Fing 9

Ratio, :ChR-ChI/XR-XI

Author: Koury and Epker

Target $: 0.4$

\section{late- II}

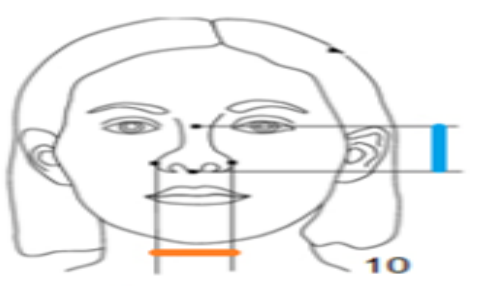

Fig 10

Ratio 10 : AIR-

AII/N-Sn

Author: Koury and

Epker

Target $: 0.625$
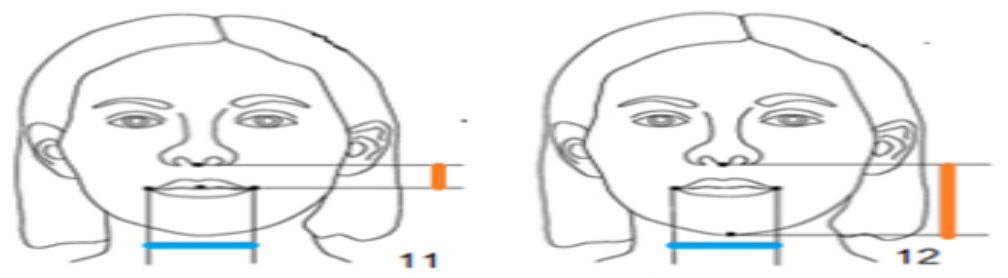

Fig 11

Ratio 11 :Sn-St/ ChR-ChI

Author: Farkas et al

Target $=0.4$

Fig 12

Ratio 12 :Sn-

Me/ChR-ChI

Author: Farkas et al

Target $=1.33$
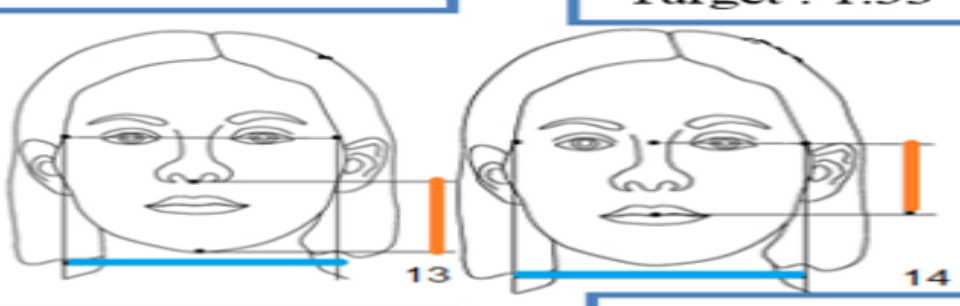

Fig 14

Ratio 14 : N-

St/XR-XI

Author: Farkas et al

Target : 0.535

Ratio 13 :Sn-Me/ $\mathrm{Xr}$

Target $=0.53$

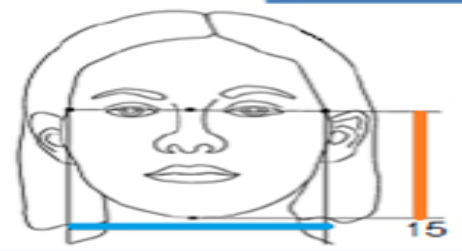

Fig 15

Ratio 15 : N-Me/XR-XI

Author: Farkas et al

Target : 0.86 


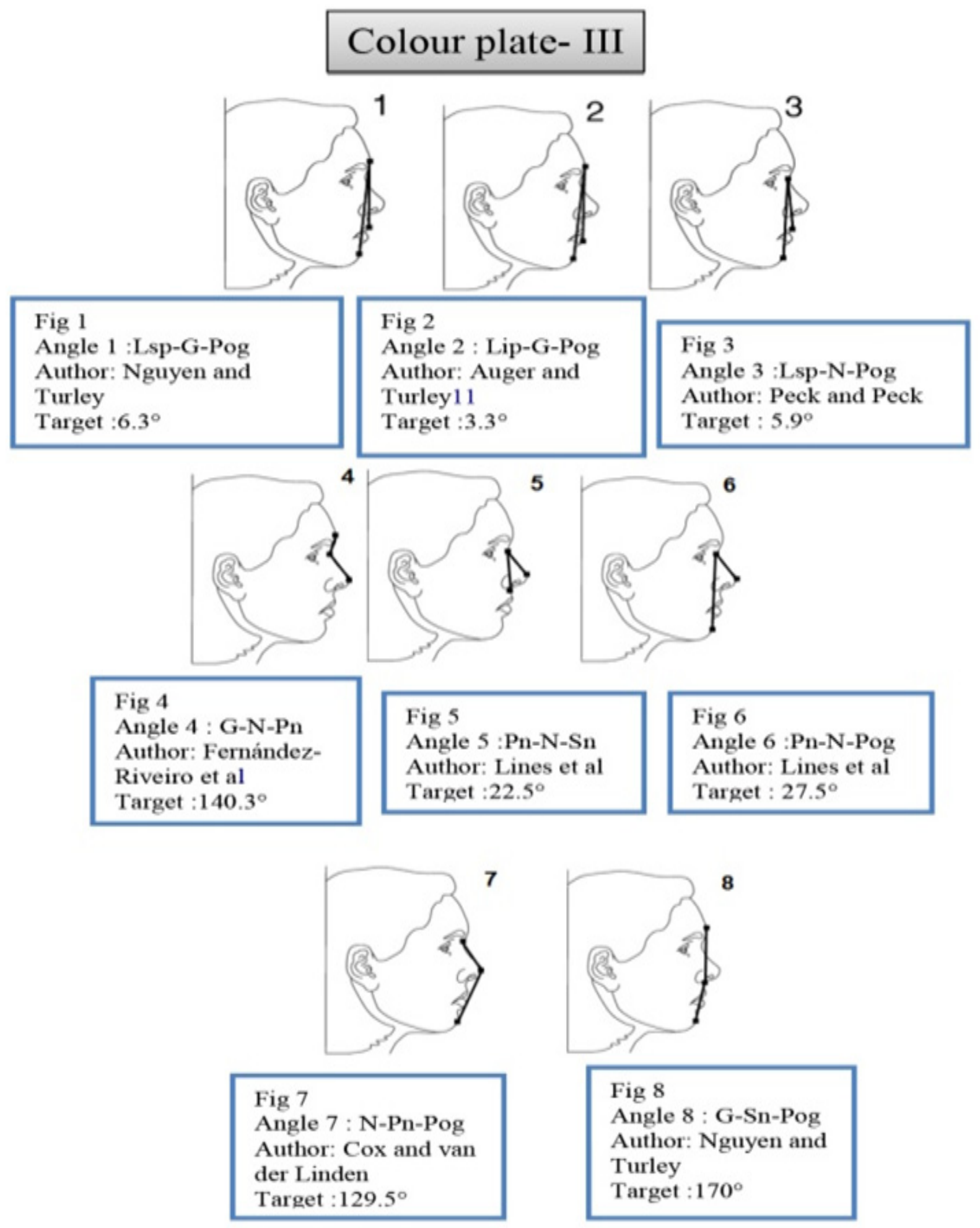




\section{Colour plate- IV}

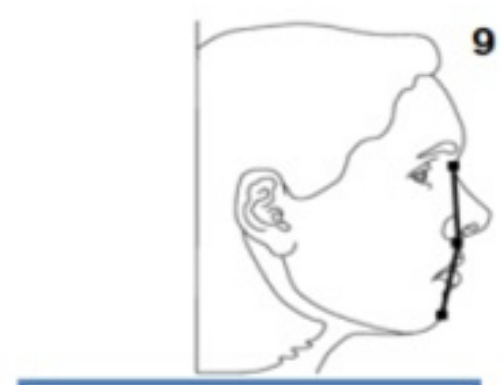

Fig 9

Angle 9 : N-Sn-Pog

Author: Cox and van

der Linden

Target : $163^{\circ}$
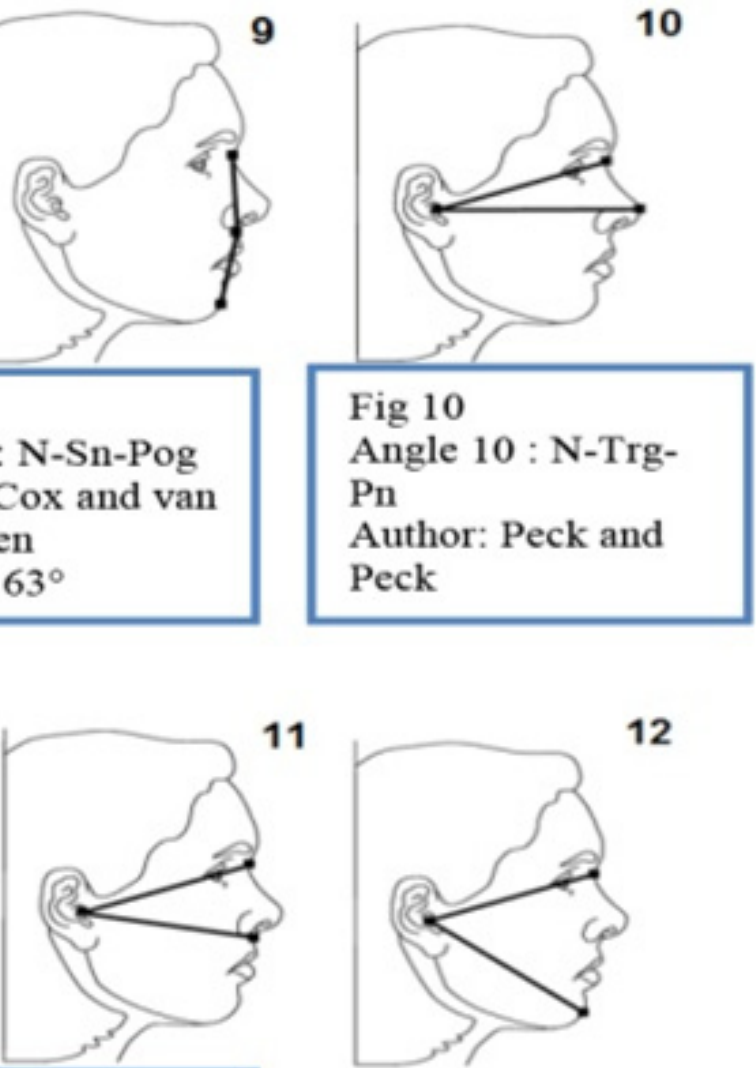

11

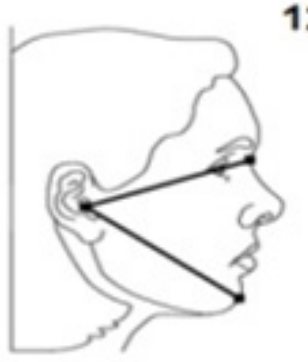

Fig 11

Angle $11: \mathrm{N}-\mathrm{Trg}-\mathrm{Sn}$

Author: Fernández-

Riveiro et al

Target : $28.5^{\circ}$

Fig 12

Angle $12: \mathrm{N}-\mathrm{Trg}-\mathrm{Pog}$ Author: Peck and Peck Target $: 54.4^{\circ}$

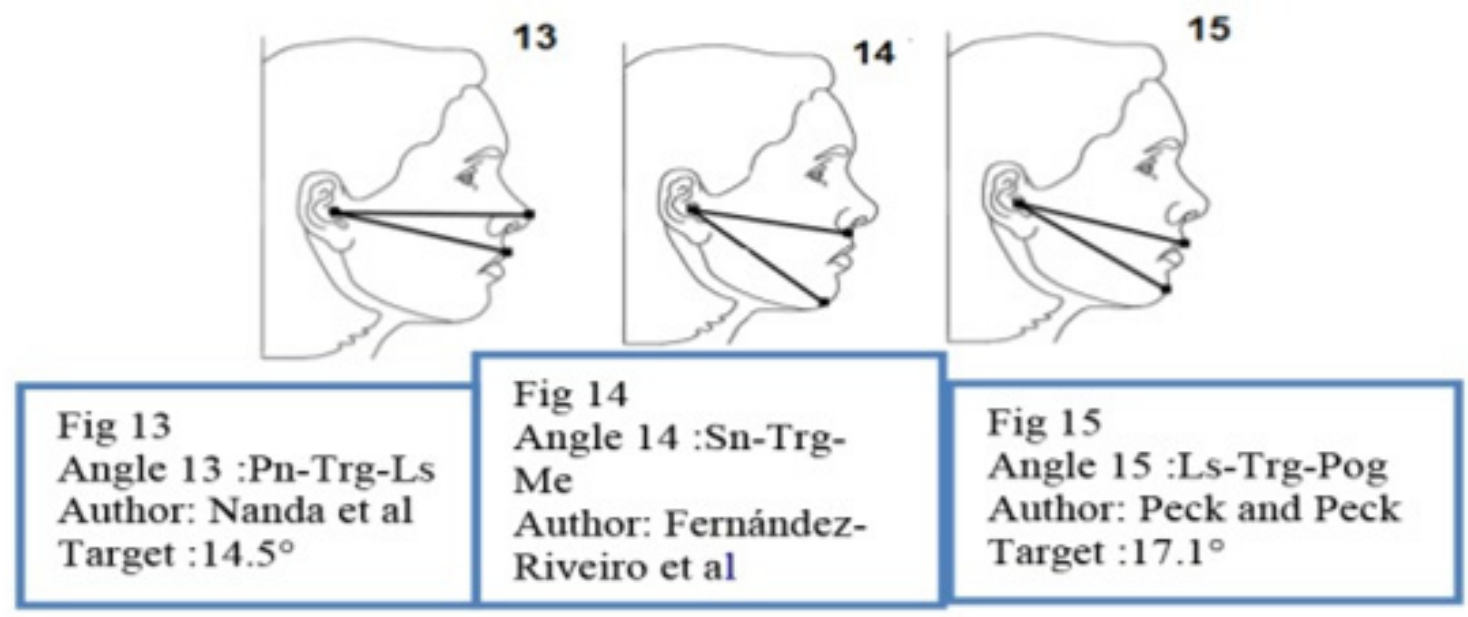


Table 1 : Descriptive Statistics for 15 Investigated Ratios on Frontal photographs

\begin{tabular}{|c|c|c|c|c|c|c|c|c|c|}
\hline S.No & Variable & Description & Gender & $\mathbf{N}$ & Mean & $\begin{array}{c}\text { Std } \\
\text { Deviation }\end{array}$ & $\begin{array}{c}\text { Std } \\
\text { Error }\end{array}$ & Min & Max \\
\hline 1. & Ratio 1 & Tr-N/N-St & \begin{tabular}{r|} 
Male \\
Female
\end{tabular} & $\begin{array}{l}40 \\
40\end{array}$ & $\begin{array}{l}1.0225 \\
1.1315\end{array}$ & $\begin{array}{l}.09021 \\
.06833\end{array}$ & $\begin{array}{l}.01426 \\
.01080\end{array}$ & \begin{tabular}{l|}
.83 \\
.97
\end{tabular} & $\begin{array}{l}1.19 \\
1.30\end{array}$ \\
\hline 2. & Ratio 2 & Tr-N/Sn-Me & $\begin{array}{r}\text { Male } \\
\text { Female }\end{array}$ & $\begin{array}{l}40 \\
40\end{array}$ & \begin{tabular}{l|}
1.0150 \\
1.2195
\end{tabular} & $\begin{array}{l}.10476 \\
.14308\end{array}$ & $\begin{array}{l}.01656 \\
.02262\end{array}$ & \begin{tabular}{r|}
.78 \\
1.00
\end{tabular} & $\begin{array}{l}1.30 \\
1.90\end{array}$ \\
\hline 3. & Ratio 3 & N-St/Sn-Me & $\begin{array}{r}\text { Male } \\
\text { Female }\end{array}$ & $\begin{array}{l}40 \\
40\end{array}$ & $\begin{array}{r}.9900 \\
1.0468\end{array}$ & $\begin{array}{l}.05706 \\
.06870\end{array}$ & $\begin{array}{l}.00902 \\
.01086\end{array}$ & $\begin{array}{l}.88 \\
.90\end{array}$ & $\begin{array}{l}1.11 \\
1.21\end{array}$ \\
\hline 4. & Ratio 4 & $\mathrm{Sn}-\mathrm{St} / \mathrm{Sn}-\mathrm{Me}$ & $\begin{array}{r}\text { Male } \\
\text { Female }\end{array}$ & $\begin{array}{l}40 \\
40\end{array}$ & $\begin{array}{l}.3135 \\
.3150\end{array}$ & $\begin{array}{l}.02020 \\
.02172\end{array}$ & $\begin{array}{l}.00319 \\
.00343\end{array}$ & $\begin{array}{l}.27 \\
.28\end{array}$ & $\begin{array}{l}.35 \\
.35\end{array}$ \\
\hline 5. & Ratio 5 & St-Me/Sn-Me & \begin{tabular}{r|} 
Male \\
Female
\end{tabular} & \begin{tabular}{l|}
40 \\
40
\end{tabular} & $\begin{array}{l}.6738 \\
.6695\end{array}$ & $\begin{array}{l}.03927 \\
.03623\end{array}$ & $\begin{array}{l}.00621 \\
.00573\end{array}$ & $\begin{array}{l}.47 \\
.51\end{array}$ & $\begin{array}{l}.75 \\
.73\end{array}$ \\
\hline 6. & Ratio 6 & Sn-St/St-Me & $\begin{array}{r}\text { Male } \\
\text { Female }\end{array}$ & $\begin{array}{l}40 \\
40\end{array}$ & $\begin{array}{l}.4607 \\
.4752\end{array}$ & $\begin{array}{l}.03938 \\
.04750\end{array}$ & $\begin{array}{l}.00623 \\
.00751\end{array}$ & $\begin{array}{l}.36 \\
.40\end{array}$ & $\begin{array}{l}.52 \\
.57\end{array}$ \\
\hline 7. & Ratio 7 & Ls-St/St-Li & $\begin{array}{r}\text { Male } \\
\text { Female }\end{array}$ & $\begin{array}{l}40 \\
40\end{array}$ & $\begin{array}{l}.6928 \\
.6363\end{array}$ & $\begin{array}{l}.14040 \\
.11593\end{array}$ & $\begin{array}{l}.02220 \\
.01833\end{array}$ & $\begin{array}{l}.29 \\
.33\end{array}$ & $\begin{array}{r}1.00 \\
.86\end{array}$ \\
\hline 8. & Ratio 8 & AIR-AIL/ChR-ChL & $\begin{array}{r}\text { Male } \\
\text { Female }\end{array}$ & $\begin{array}{l}40 \\
40\end{array}$ & $\begin{array}{l}.7962 \\
.7617\end{array}$ & $\begin{array}{l}.04980 \\
.11003\end{array}$ & $\begin{array}{l}.00787 \\
.01740\end{array}$ & $\begin{array}{l}.70 \\
.63\end{array}$ & $\begin{array}{r}.90 \\
1.29\end{array}$ \\
\hline 9. & Ratio 9 & ChR-ChL/XR-XL & \begin{tabular}{r|} 
Male \\
Female
\end{tabular} & $\begin{array}{l}40 \\
40\end{array}$ & $\begin{array}{l}.3585 \\
.3672\end{array}$ & $\begin{array}{l}.02214 \\
.03493\end{array}$ & $\begin{array}{l}.00350 \\
.00552\end{array}$ & $\begin{array}{l}.32 \\
.26\end{array}$ & $\begin{array}{l}.41 \\
.43\end{array}$ \\
\hline 10. & Ratio 10 & AIR-AIL/N-Sn & \begin{tabular}{r|} 
Male \\
Female
\end{tabular} & $\begin{array}{l}40 \\
40\end{array}$ & $\begin{array}{l}.8830 \\
.8325\end{array}$ & $\begin{array}{l}.09365 \\
.08073\end{array}$ & $\begin{array}{l}.01481 \\
.01276\end{array}$ & $\begin{array}{l}.60 \\
.62\end{array}$ & $\begin{array}{l}1.05 \\
1.03\end{array}$ \\
\hline 11. & Ratio 11 & Sn-St/ ChR-ChL & \begin{tabular}{r|} 
Male \\
Female
\end{tabular} & $\begin{array}{l}40 \\
40\end{array}$ & $\begin{array}{l}.4238 \\
.3900\end{array}$ & $\begin{array}{l}.03801 \\
.04997\end{array}$ & $\begin{array}{l}.00601 \\
.00790\end{array}$ & $\begin{array}{l}.35 \\
.26\end{array}$ & $\begin{array}{l}.50 \\
.48\end{array}$ \\
\hline 12. & Ratio 12 & Sn-Me/ChR-ChL & \begin{tabular}{r|} 
Male \\
Female
\end{tabular} & $\begin{array}{l}40 \\
40\end{array}$ & \begin{tabular}{l|}
1.341 \\
1.233
\end{tabular} & $\begin{array}{l}.0969 \\
.1280\end{array}$ & $\begin{array}{l}.0153 \\
.0202\end{array}$ & $\begin{array}{r}1.2 \\
.9\end{array}$ & $\begin{array}{l}1.6 \\
1.5\end{array}$ \\
\hline 13. & Ratio 13 & Sn-Me/XR-XL & $\begin{array}{r}\text { Male } \\
\text { Female }\end{array}$ & $\begin{array}{l}40 \\
40\end{array}$ & \begin{tabular}{l|}
.4827 \\
.4533
\end{tabular} & $\begin{array}{l}.02918 \\
.02982\end{array}$ & $\begin{array}{l}.00461 \\
.00471\end{array}$ & \begin{tabular}{l|}
.43 \\
.39
\end{tabular} & $\begin{array}{l}.54 \\
.53\end{array}$ \\
\hline 14. & Ratio 14 & N-St/XR-XL & $\begin{array}{r}\text { Male } \\
\text { Female }\end{array}$ & $\begin{array}{l}40 \\
40\end{array}$ & $\begin{array}{l}.4815 \\
.4698\end{array}$ & $\begin{array}{l}.02095 \\
.02966\end{array}$ & $\begin{array}{l}.00331 \\
.00469\end{array}$ & $\begin{array}{l}.42 \\
.33\end{array}$ & $\begin{array}{l}.53 \\
.52\end{array}$ \\
\hline 15. & Ratio 15 & N-Me/XR-XL & $\begin{array}{r}\text { Male } \\
\text { Female }\end{array}$ & $\begin{array}{l}40 \\
40\end{array}$ & $\begin{array}{l}.8112 \\
.7808\end{array}$ & $\begin{array}{l}.02875 \\
.02921\end{array}$ & $\begin{array}{l}.00455 \\
.00462\end{array}$ & $\begin{array}{l}.76 \\
.71\end{array}$ & $\begin{array}{l}.90 \\
.87\end{array}$ \\
\hline
\end{tabular}


Table 2 : Descriptive Statistics for 15 Investigated Angles on Lateral Photographs

\begin{tabular}{|c|c|c|c|c|c|c|c|c|c|}
\hline S.No & Variable & Description & Gender & $\mathbf{N}$ & Mean & $\begin{array}{c}\text { Std } \\
\text { Deviation }\end{array}$ & $\begin{array}{c}\text { Std } \\
\text { Error }\end{array}$ & Min & Max \\
\hline \multirow[t]{2}{*}{1.} & Angle 1 & Lsp-G-Pog & Male & 40 & 6.15 & 1.562 & .247 & 3 & 9 \\
\hline & & & Female & 40 & 5.93 & 1.207 & .191 & 3 & 8 \\
\hline \multirow[t]{2}{*}{2.} & Angle 2 & Lip-G-Pog & Male & 40 & 3.38 & 1.192 & .188 & 1 & 6 \\
\hline & & & Female & 40 & 3.68 & 1.141 & .180 & 2 & 7 \\
\hline \multirow[t]{2}{*}{3.} & Angle 3 & Lsp-N-Pog & Male & 40 & 8.88 & 1.897 & .300 & 5 & 13 \\
\hline & & & Female & 40 & 8.43 & 1.583 & .250 & 4 & 11 \\
\hline \multirow[t]{2}{*}{4.} & Angle 4 & G-N-Pn & Male & 40 & 130.53 & 7.699 & 1.217 & 115 & 152 \\
\hline & & & Female & 40 & 133.05 & 5.439 & .860 & 118 & 147 \\
\hline \multirow[t]{2}{*}{5.} & Angle 5 & Pn-N-Sn & Male & 40 & 20.90 & 2.318 & .367 & 17 & 26 \\
\hline & & & Female & 40 & 20.00 & 1.908 & .302 & 16 & 23 \\
\hline \multirow[t]{2}{*}{6.} & Angle 6 & Pn-N-Pog & Male & 40 & 31.10 & 2.951 & .467 & 25 & 37 \\
\hline & & & Female & 40 & 29.73 & 2.276 & .360 & 25 & 34 \\
\hline \multirow[t]{2}{*}{7.} & Angle 7 & N-Pn-Pog & Male & 40 & 129.35 & 3.620 & .572 & 122 & 137 \\
\hline & & & Female & 40 & 127.23 & 8.862 & 2.982 & 131 & 141 \\
\hline \multirow[t]{2}{*}{8.} & Angle 8 & G-Sn-Pog & Male & 40 & 166.80 & 4.220 & .667 & 157 & 175 \\
\hline & & & Female & 40 & 166.30 & 4.256 & .673 & 157 & 175 \\
\hline \multirow[t]{2}{*}{9.} & Angle 9 & N-Sn-Pog & Male & 40 & 160.38 & 3.946 & .624 & 153 & 168 \\
\hline & & & Female & 40 & 160.48 & 4.723 & .747 & 151 & 170 \\
\hline \multirow[t]{2}{*}{10.} & Angle 10 & N-Trg-Pn & Male & 40 & 19.93 & 1.789 & .283 & 17 & 24 \\
\hline & & & Female & 40 & 20.25 & 1.891 & .299 & 16 & 24 \\
\hline \multirow[t]{2}{*}{11.} & Angle 11 & N-Trg-Sn & Male & 40 & 26.28 & 1.783 & .282 & 22 & 30 \\
\hline & & & Female & 40 & 26.65 & 2.315 & .366 & 23 & 32 \\
\hline \multirow[t]{2}{*}{12.} & Angle 12 & N-Trg-Pog & Male & 40 & 50.83 & 3.129 & .495 & 44 & 57 \\
\hline & & & Female & 40 & 49.80 & 3.488 & .551 & 43 & 59 \\
\hline \multirow[t]{2}{*}{13.} & Angle 13 & Pn-Trg-Ls & Male & 40 & 12.13 & 1.505 & .238 & 10 & 16 \\
\hline & & & Female & 40 & 11.75 & 1.296 & .205 & 10 & 14 \\
\hline \multirow[t]{2}{*}{14.} & Angle 14 & Sn-Trg-Me & Male & 40 & 28.70 & 3.139 & .496 & 29 & 34 \\
\hline & & & Female & 40 & 28.15 & 2.119 & .335 & 27 & 34 \\
\hline \multirow[t]{2}{*}{15.} & Angle 15 & Ls-Trg-Pog & Male & 40 & 19.25 & 2.509 & .397 & 15 & 30 \\
\hline & & & Female & 40 & 18.20 & 1.757 & .278 & 14 & 22 \\
\hline
\end{tabular}


Table 3 :

Frontal photographic Analysis: Comparison of Males \& Females of PRESENT Study

\begin{tabular}{|c|c|c|c|c|c|c|c|}
\hline \multirow[t]{2}{*}{ S No } & \multirow[t]{2}{*}{ Variable } & \multirow[t]{2}{*}{ Description } & \multicolumn{2}{|c|}{ Males } & \multicolumn{2}{|c|}{ Females } & \multirow[t]{2}{*}{$\begin{array}{l}\text { P value } \\
\text { (Significance) }\end{array}$} \\
\hline & & & Mean & SD & Mean & SD & \\
\hline 1 & Ratio 1 & Tr-N/N-St & 1.02 & 0.09 & 1.13 & 0.07 & 0.00 * \\
\hline 2 & Ratio 2 & Tr-N/Sn-Me & 1.01 & 0.10 & 1.22 & 0.14 & $0.00 *$ \\
\hline 3 & Ratio 3 & $\mathrm{~N}-\mathrm{St} / \mathrm{Sn}-\mathrm{Me}$ & 0.99 & 0.06 & 1.04 & 0.07 & 0.00 * \\
\hline 4 & Ratio 4 & Sn-St/Sn-Me & 0.31 & 0.02 & 0.31 & 0.02 & 0.75 \\
\hline 5 & Ratio 5 & St-Me/Sn-Me & 0.67 & 0.04 & 0.67 & 0.04 & 0.61 \\
\hline 6 & Ratio 6 & Sn-St/St-Me & 0.46 & 0.04 & 0.47 & 0.05 & 0.14 \\
\hline 7 & Ratio 7 & Ls-St/St-Li & 0.69 & 0.14 & 0.64 & 0.11 & $0.05 *$ \\
\hline 8 & Ratio 8 & AIR-AIL/ChR-ChL & 0.79 & 0.05 & 0.76 & 0.11 & 0.07 \\
\hline 9 & Ratio 9 & ChR-ChL/ XR-XL & 0.36 & 0.02 & 0.37 & 0.03 & 0.18 \\
\hline 10 & Ratio 10 & AIR-AIL/N-Sn & 0.88 & 0.09 & 0.83 & 0.08 & $0.01 *$ \\
\hline 11 & Ratio 11 & $\mathrm{Sn}-\mathrm{St} / \mathrm{ChR}-\mathrm{ChL}$ & 0.42 & 0.04 & 0.39 & 0.05 & 0.00 * \\
\hline 12 & Ratio 12 & Sn-Me/ChR-ChL & 1.34 & 0.10 & 1.23 & 0.13 & 0.00 * \\
\hline 13 & Ratio 13 & Sn-Me/XR-XL & 0.48 & 0.03 & 0.45 & 0.03 & $0.00 *$ \\
\hline 14 & Ratio 14 & N-St/XR-XL & 0.48 & 0.02 & 0.47 & 0.03 & 0.04 * \\
\hline 15 & Ratio 15 & N-Me/XR-XL & 0.81 & 0.03 & 0.78 & 0.03 & $0.00 *$ \\
\hline
\end{tabular}

*Significant at $P<0.05$ 
Table 4 :

Lateral photographic Analysis: Comparison of Males \& Females of PRESENT Study

\begin{tabular}{|c|c|c|c|c|c|c|c|}
\hline \multirow{2}{*}{ S No } & \multirow{2}{*}{ Variable } & \multirow{2}{*}{ Description } & \multicolumn{2}{|c|}{ Males } & \multicolumn{2}{|c|}{ Females } & \multirow{2}{*}{$\begin{array}{l}\text { P value } \\
\text { (Significance) }\end{array}$} \\
\hline & & & Mean & SD & Mean & SD & \\
\hline 1. & Angle 1 & Lsp-G-Pog & 6.15 & 1.56 & 5.93 & 1.20 & 0.47 \\
\hline 2. & Angle 2 & Lip-G-Pog & 3.38 & 1.20 & 3.68 & 1.14 & 0.25 \\
\hline 3. & Angle 3 & Lsp-N-Pog & 8.88 & 1.90 & 8.43 & 1.58 & 0.25 \\
\hline 4. & Angle 4 & G-N-Prn & 130.53 & 7.70 & 133.05 & 5.44 & 0.09 \\
\hline 5. & Angle 5 & Prn-N-Sn & 20.90 & 2.31 & 20.00 & 1.90 & 0.06 \\
\hline 6. & Angle 6 & Prn-N-Pog & 31.10 & 2.95 & 29.73 & 2.27 & $0.02 *$ \\
\hline 7. & Angle 7 & N-Prn-Pog & 129.35 & 3.62 & 127.23 & 8.86 & 0.48 \\
\hline 8. & Angle 8 & G-Sn-Pog & 166.80 & 4.22 & 166.30 & 4.26 & 0.60 \\
\hline 9. & Angle 9 & N-Sn-Pog & 160.38 & 3.95 & 160.48 & 4.72 & 0.91 \\
\hline 10. & Angle 10 & N-Trg-Prn & 19.93 & 1.79 & 20.25 & 1.89 & 0.43 \\
\hline 11. & Angle 11 & N-Trg-Sn & 26.28 & 1.78 & 26.65 & 2.31 & 0.42 \\
\hline 12. & Angle 12 & N-Trg-Pog & 50.83 & 3.12 & 49.8 & 3.49 & 0.17 \\
\hline 13. & Angle 13 & Prn-Trg-Ls & 12.13 & 1.50 & 11.75 & 1.29 & 0.23 \\
\hline 14. & Angle 14 & Sn-Trg-Me & 28.7 & 3.13 & 28.15 & 2.11 & 0.36 \\
\hline 15. & Angle 15 & Ls-Trg-Pog & 19.25 & 2.50 & 18.20 & 1.76 & $0.03 *$ \\
\hline
\end{tabular}

$*$ Significant at $P<0.05$ 
Table 5:

Frontal photographic Analysis: Comparison of Hyderabad and Caucasian Male Sample

\begin{tabular}{|c|c|c|c|c|c|}
\hline S No & Variable & Description & $\begin{array}{l}\text { Indian } \\
\text { Mean }\end{array}$ & $\begin{array}{l}\text { Caucasian } \\
\text { mean }\end{array}$ & P value \\
\hline 1. & Ratio 1 & Tr-N/N-St & 1.02 & 1 & 0.12 \\
\hline 2. & Ratio 2 & Tr-N/Sn-Me & 1.01 & 1 & 0.37 \\
\hline 3. & Ratio 3 & $\mathrm{~N}-\mathrm{St} / \mathrm{Sn}-\mathrm{Me}$ & 0.99 & 1 & 0.27 \\
\hline 4. & Ratio 4 & $\mathrm{Sn}-\mathrm{St} / \mathrm{Sn}-\mathrm{Me}$ & 0.31 & 0.32 & 0.10 \\
\hline 5. & Ratio 5 & St-Me/Sn-Me & 0.67 & 0.66 & 0.20 \\
\hline 6. & Ratio 6 & Sn-St/St-Me & 0.46 & 0.5 & $0.00 *$ \\
\hline 7. & Ratio 7 & Ls-St/St-Li & 0.69 & 0.97 & $0.00 *$ \\
\hline 8. & Ratio 8 & AIR-AIL/ChR-ChL & 0.79 & 0.63 & $0.00 *$ \\
\hline 9. & Ratio 9 & ChR-ChL/ XR-XL & 0.36 & 0.38 & $0.00 *$ \\
\hline 10. & Ratio 10 & AIR-AIL/N-Sn & 0.88 & 0.63 & $0.00 *$ \\
\hline 11. & Ratio 11 & Sn-St/ChR-ChL & 0.42 & 0.41 & 0.28 \\
\hline 12. & Ratio 12 & Sn-Me/ChR-ChL & 1.34 & 1.33 & 0.40 \\
\hline 13. & Ratio 13 & Sn-Me/XR-XL & 0.48 & 0.53 & $0.00 *$ \\
\hline 14. & Ratio 14 & N-St/XR-XL & 0.48 & 0.54 & $0.00 *$ \\
\hline 15. & Ratio 15 & N-Me/XR-XL & 0.81 & 0.88 & $0.00 *$ \\
\hline
\end{tabular}

$*$ Significant at $P<0.05$ 
Table 6 :

Frontal photographic Analysis: Comparison of Hyderabad and Caucasian Female Sample

\begin{tabular}{|c|c|c|c|c|c|}
\hline S No & Variable & Description & $\begin{array}{l}\text { Indian } \\
\text { Mean }\end{array}$ & $\begin{array}{l}\text { Caucasian } \\
\text { mean }\end{array}$ & $P$ value \\
\hline 1. & Ratio 1 & Tr-N/N-St & 1.13 & 1 & $0.00 *$ \\
\hline 2. & Ratio 2 & Tr-N/Sn-Me & 1.21 & 1 & $0.00 *$ \\
\hline 3. & Ratio 3 & $\mathrm{~N}-\mathrm{St} / \mathrm{Sn}-\mathrm{Me}$ & 1.04 & 1 & $0.00 *$ \\
\hline 4. & Ratio 4 & $\mathrm{Sn}-\mathrm{St} / \mathrm{Sn}-\mathrm{Me}$ & 0.31 & 0.31 & 0.25 \\
\hline 5. & Ratio 5 & $\mathrm{St}-\mathrm{Me} / \mathrm{Sn}-\mathrm{Me}$ & 0.66 & 0.66 & 0.66 \\
\hline 6. & Ratio 6 & Sn-St/St-Me & 0.47 & 0.5 & $0.02 *$ \\
\hline 7. & Ratio 7 & Ls-St/St-Li & 0.63 & 0.87 & $0.00 *$ \\
\hline 8. & Ratio 8 & AIR-AIL/ChR-ChL & 0.76 & 0.61 & $0.00 \%$ \\
\hline 9. & Ratio 9 & ChR-ChL/XR-XL & 0.36 & 0.38 & $0.00 *$ \\
\hline 10. & Ratio 10 & AIR-AIL/N-Sn & 0.83 & 0.61 & $0.00 *$ \\
\hline 11. & Ratio 11 & Sn-St/ChR-ChL & 0.39 & 0.39 & 1 \\
\hline 12. & Ratio 12 & $\mathrm{Sn}-\mathrm{Me} / \mathrm{ChR}-\mathrm{ChL}$ & 1.33 & 1.33 & 0.56 \\
\hline 13. & Ratio 13 & Sn-Me/XR-XL & 0.45 & 0.53 & $0.00 *$ \\
\hline 14. & Ratio 14 & N-St/XR-XL & 0.46 & 0.52 & $0.00 *$ \\
\hline 15. & Ratio 15 & N-Me/XR-XL & 0.78 & 0.86 & $0.00 \%$ \\
\hline
\end{tabular}

*Significant at $P<0.05$ 
Table 7:

Lateral photographic Analysis: Comparison of Hyderabad and Caucasian Male Sample

\begin{tabular}{|c|c|c|c|c|c|}
\hline S No & Variable & Description & $\begin{array}{l}\text { Indian } \\
\text { Mean }\end{array}$ & $\begin{array}{l}\text { Caucasian } \\
\text { mean }\end{array}$ & P value \\
\hline 1. & Angle 1 & Lsp-G-Pog & 6.15 & 4.7 & $0.04 *$ \\
\hline 2. & Angle 2 & Lip-G-Pog & 3.38 & 2.2 & $0.02 *$ \\
\hline 3. & Angle 3 & Lsp-N-Pog & 8.88 & 5.9 & $0.00 *$ \\
\hline 4. & Angle 4 & G-N-Prn & 130.53 & 138.6 & $0.00 *$ \\
\hline 5. & Angle 5 & Prn-N-Sn & 20.90 & 22.5 & $0.00 *$ \\
\hline 6. & Angle 6 & Prn-N-Pog & 31.10 & 27.5 & $0.00 *$ \\
\hline 7. & Angle 7 & N-Prn-Pog & 129.35 & 129.5 & 0.4 \\
\hline 8. & Angle 8 & G-Sn-Pog & 166.80 & 168.2 & $0.00 *$ \\
\hline 9. & Angle 9 & N-Sn-Pog & 160.38 & 163 & $0.00 *$ \\
\hline 10. & Angle 10 & N-Trg-Prn & 19.93 & 23.6 & $0.00 \%$ \\
\hline 11. & Angle 11 & N-Trg-Sn & 26.28 & 28.9 & $0.00 *$ \\
\hline 12. & Angle 12 & N-Trg-Pog & 50.83 & 54.5 & $0.00 *$ \\
\hline 13. & Angle 13 & Prn-Trg-Ls & 12.13 & 14.1 & $0.00 *$ \\
\hline 14. & Angle 14 & Sn-Trg-Me & 28.7 & 36.8 & $0.00^{*}$ \\
\hline 15. & Angle 15 & Ls-Trg-Pog & 19.25 & 17.1 & 0.00* \\
\hline
\end{tabular}

$*$ Significant at $P<0.05$ 
Table 8:

Lateral photographic Analysis: Comparison of Hyderabad and Caucasian Female Sample

\begin{tabular}{|c|c|c|c|c|c|}
\hline S No & Variable & Description & $\begin{array}{l}\text { Indian } \\
\text { Mean }\end{array}$ & $\begin{array}{l}\text { Caucasian } \\
\text { mean }\end{array}$ & P value \\
\hline 1. & Angle 1 & Lsp-G-Pog & 5.93 & 6.3 & $0.04 *$ \\
\hline 2. & Angle 2 & Lip-G-Pog & 3.68 & 3.3 & $0.02 *$ \\
\hline 3. & Angle 3 & Lsp-N-Pog & 8.43 & 5.9 & $0.00 *$ \\
\hline 4. & Angle 4 & G-N-Prn & 133.05 & 141.9 & $0.00 *$ \\
\hline 5. & Angle 5 & Prn-N-Sn & 20.00 & 22.5 & $0.00 *$ \\
\hline 6. & Angle 6 & Prn-N-Pog & 29.73 & 27.5 & $0.00 *$ \\
\hline 7. & Angle 7 & N-Prn-Pog & 127.23 & 129.5 & 0.7 \\
\hline 8. & Angle 8 & G-Sn-Pog & 166.30 & 167 & $0.00 *$ \\
\hline 9. & Angle 9 & N-Sn-Pog & 160.48 & 163 & $0.00 *$ \\
\hline 10. & Angle 10 & N-Trg-Prn & 20.25 & 23.6 & $0.00 *$ \\
\hline 11. & Angle 11 & N-Trg-Sn & 26.65 & 28.2 & $0.00 *$ \\
\hline 12. & Angle 12 & N-Trg-Pog & 49.8 & 54.5 & $0.00 *$ \\
\hline 13. & Angle 13 & Prn-Trg-Ls & 11.75 & 14.9 & $0.00 *$ \\
\hline 14. & Angle 14 & Sn-Trg-Me & 28.15 & 36.2 & $0.00 *$ \\
\hline 15. & Angle 15 & Ls-Trg-Pog & 18.20 & 17.1 & $0.00 *$ \\
\hline
\end{tabular}

$*$ Significant at $P<0.05$ 
plate I) males $=0.67 \pm 0.04$, females $=0.67 \pm 0.04$ ) was similar for Hyderabad males and females and no difference was seen between Hyderabad population and Caucasians.

Upper to lower lip ratio (Sn-St/St-Me, (F igure 6, colour plate I) males $=0.46 \pm 0.04$, females $=0.47 \pm 0.05)$ significantly decreased $(P=0.00)$, indicating increased lower lip length. Arnett and Bergman (1993a, b) presented a clinical facial analysis based on previous studies and their surgical experience. ${ }^{15,16}$ According to the authors, the normal ratio of upper to lower lip is 1:2 (0.5). Proportionate lips harmonize regardless of length. Vermilion height Index, (Figure 7, colour plate I) (Ls-St/ St-Li, $\mathrm{P}=0.00$ ) also significantly decreased in our population, indicating increased lower vermilion height. Significant sexual dimorphism was also noted (Ls-St/St-Li,males $=0.69 \pm 0.14$, females $=0.64 \pm 0.11, P=0.05)$. The Hyderabad females displayed a decreased ratio over the males, possibly due to lower vermilion height.

Nose-Mouth width Index, (Figure 8, colour plate I) (AIR-AIL/ChR-ChL, males $=0.79 \pm 0.05$, females $=0.76 \pm 0.11$ ) values of males and females were al most the same, and were significantly more than the Caucasian ratio (AIR-AIL/ChR-ChL, $P=0.00$ ) indicating increased width of the alar base. This is in accordance with Sarver's observation that "the width of the alar base is heavily influenced by inherited ethnic characteristics. ${ }^{17}$ It is very important to be sensitive to the ethnicity of the patient when variations in nasal proportions are discussed.

Nasal Index (Figure 10, colour plate II) showed significant sexual dimorphism (AIR-AIL/NSn, males $=0.88 \pm 0.09$, females $=0.83 \pm 0.08, P=0.01$ ). The Hyderabad females displayed a decreased ratio over the males, possibly due to the greater distance from nasion to subnasale in females. When Hyderabad and Caucasian values were compared, the mean difference was significant.

U pper face height -M outh width Index (Figure 11, colour plate II) (Sn-St/ChR-ChL, males= $0.42 \pm 0.04$, females $=0.39 \pm 0.05, P=0.00$ ) and Lower face height- Mouth width index (Figure 12, colour plate II) $(\mathrm{Sn}-\mathrm{Me} / \mathrm{ChR}-\mathrm{ChL}$, males $=1.34 \pm 0.10$, females $=1.23 \pm 0.13, P=0.00$ ) showed significant sexual differences. Females displayed decreased ratio over the males, probably due to decreased width of the mouth when compared to males and no difference was seen between us and Caucasians. Mouth seems to be wider in men than in women. The Lower face height-Face width Index (Figure 13, colour plate II; Sn-Me/XR-XL, males = $0.48 \pm 0.03$, females $=0.45 \pm 0.03, P=0.00$ ) and The U pper face height-Face width Index (Figure 14, colour plate II; N-St/XR-XL, males $=0.48 \pm 0.02$, females $=0.46 \pm 0.03, P=0.04$ ) showed significant sexual differences in our population. Females showed slightly larger lower face height than males. On comparing Hyderabad and Caucasian values, the Caucasian values were significantly greater indicating that Caucasians have relatively large lower half of the face.

The proportional relationship of facial height to width (the facial index, Figure 15, colour plate II), more than the absolute value of either, establish overall facial type and the basic proportions of the face. This measurement relates the vertical dimension of the face to horizontal dimension. The facial index (N-Me/XR-XL, males $=0.81 \pm 0.03$, females $=0.78 \pm 0.03, P=0.00$ ) showed significant sexual differences in our population. On comparing Indian and Caucasian values, the Caucasian values were significantly greater ( $\mathrm{N}-\mathrm{M}$ e/XR-XL, $\mathrm{P}=0.00$ ) indicating that the facial index was greater inthe Caucasian population. This could be on account of racial differences.

\section{Lateral Photographic Analysis:}

Upper Lip Projection (Lsp-G-Pog; (Figure 1, colour plate III); males $=6.15 \pm 1.6$ degrees, females $=5.93 \pm 1.2$ degrees) and Lower Lip Projection (Lip-G-Pog; (Figure 2, colour plate III; males $=3.38 \pm 1.2$ degrees, females $=3.68 \pm 1.1$ degrees.) showed statistically significant differences between Hyderabad and Caucasian population (Lsp-G-Pog, $P=0.05$; Lip-G-Pog, $P=0.04$ ). Both angular measurements were significantly more than the Caucasian values. Males showed more inclination of upper lips from vertical than female population and females showed more inclination of lower lips from vertical than male population, though not 
statistically significant. Maxillofacial angle (Lsp-NPog; (Figure 3, colour plate III; males $=8.88 \pm 1.9$ degrees, females $=8.43 \pm 1.6$ degrees.) relates the upper lip to chin, horizontally. It may be considered as soft tissue analog to cephalometric ANB introduced by Richard Riedel. ${ }^{18}$ On comparing Hyderabad and Caucasian values, the Hyderabad values were significantly greater $(P=0.00)$, indicating that the maxillofacial angle was greater in our population. This could be on account of racial differences. Peck and Peck (1970) studied standardized cephalometric and photographic records of Caucasians with pleasing faces. ${ }^{3}$ The mean value obtained for this angle according to those authors was $5.9 \pm 1.7$ degrees. The range of angle among 52 subjects was 2.5 to 9.5 degrees. The nasofrontal angle (G-N-Prn; (Figure 4, colour plate III did not show any statistically significant sexual differences (males $=130.5 \pm 7.7$ degrees, females $=133 \pm 5.4$ degrees). Fernandez-Riviero, in a study of Angular photogrammetric analysis of the soft tissue facial profile of young adult European Caucasian population showed statistically significant sexual differences $(P<0.01$ ) (males $=138$ \pm 7 degrees, females $=142 \pm 6$ degrees). Epker $(1992)^{6}$, in a study of Caucasians undertaken on frontal and lateral facial views, observed no sexual differences in this angle (130 degrees). ${ }^{10} \mathrm{Nasal}$ prominence relative to chin angle ( $\mathrm{Prn}-\mathrm{N}-\mathrm{Sn}$, Figure 5, colour plate III, males $=20.90 \pm 2.3$ degrees, females $=20 \pm 1.9$ degrees) values were similar for Hyderabad males and females, but they were significantly less than the Caucasian values $(P=0.00)$. J acques J oseph, the German father of rhinoplasty, studied modern and ancient works of art and stated that the range for esthetic nasal prominence was 23 to 37 degrees and that the ideal was 30 degrees. ${ }^{5}$ Clements noted that in most great works of art the nasal prominence averaged 30 degrees or less. Columellar length angle showed significant sexual differences (Prn-N-Pog, Figure 6, colour plate III; males $=31.1 \pm 2.9$ degrees, females $=29.7 \pm 2.3$ degrees, $\mathrm{P}=0.02$ ) .Our population showed significantly larger columellar lengths than Caucasians. Lines PA, Lines RR, and Lines CA (1978) did a study to compare the facial profile components considered desirable for males to those of females and found that the Nasal prominence relative to chin angle was 22.5 degrees with a range of 20 to 35 degrees and columellar length angle 27.5 degrees. ${ }^{5}$ Hyderabad population exhibited more convex profiles, as indicated by the increased facial convexity angle G-Sn-Pg, (Figure 8, colour plate III) as well as increased N-Sn-Pog angle (Figure 9, colour plate IV). Hyderabad females had more convex profiles as compared to males, but the difference was not significant. According to the authors, a Class I profile presented an angle range of 165-175 degrees, a Class II profile less than 165 degrees, and a Class III greater than 175 degrees. Yuen and Hiranaka (1989) reported from their Asian adolescent sample on photographic records a G-Sn$\mathrm{Pg}$ angle of $162 \pm 5$ degrees in females and $161 \pm 6$ degrees in males. ${ }^{19}$ The G-Prn-Pg angle was $135 \pm 4$ degrees in males and $135 \pm 3$ degrees in females. No sexual dimorphism was found. In the present investigation, the facial convexity and total facial convexity angles obtained were similar. G-Sn-Pg: $166.80 \pm 4.2$ degrees in males and $166.3 \pm 4.2$ degrees in females.

Peck and Peck (1970) used a profilometric analysis based on standardized cephalograms and photographs to assess the soft tissue facial profile. ${ }^{3}$ They analyzed vertical height by means of angles such as the total vertical (N-Trg-Pog, Figure 12, colour plate IV), the nasal (N-Trg-Prn, Figure 10, colour plate IV), the maxillary (Prn-Trg-Ls, Figure 13, colourplate IV), and the mandibular (Ls-Trg-Pog, Figure 15, colour plate IV) angles. The values were approximately similar for males and females in the Hyderabad population for a composite angle representing the total vertical dimension from nasion to pogonion( N-Trg-Pog, males $=50.83 \pm 3.1$ degrees, females $=49.80 \pm 3.5$ degrees). On comparing Hyderabad and Caucasian values, the Caucasian values were significantly greater, the total vertical angle had a mean value of 54.5 with a range of 47 to 62 . The nasal angle ( $N$ Trg-Prn) which measures the nasal height from nasion to pronasale demonstrated that Hyderabad males and females had the same nasal angle (males $=19.93 \pm 1.8$ degrees, females $=20.25 \pm 1.9$ degrees), which was significantly less than that of the Caucasians. The mandibular angle showed 
significant sexual differences and was significantly more than Caucasian population.

In this investigation the middle and inferior facial thirds were evaluated by the N-Trg-Sn (Figure 11, colour plate IV) and Sn-Trg-Gn (Figure 14, colour plate IV) angles respectively. The inferior third was larger (28.4 \pm 2.7 degrees) than the middle third (26.5 \pm 2 degrees). No sexual dimorphism was seen. The Hyderabad values were significantly less than the Caucasian values.

\section{Conclusion:}

The results of this study pointed to the following conclusions:

As compared to the Caucasians, Hyderabad population has a decreased facial Index. Females showed slightly larger lower face than males. The nasal dimensions are increased transversely and reduced vertically in our population when compared to Caucasians. Hyderabad population have a decreased upper lip length to lower lip length ratio, indicating a relatively short upper lip and long lower lip. Males showed moreinclination of upper lip than females. Females showed more inclination of lower lip than males. Mouth seems to be wider in males than in females. Males and females of our population showed fuller profile and lips as compared to the Caucasian population.

Many values of our population indices did not show any correlations with the values of previously established other population indices. In conclusion, we recommend the utilization of Hyderabad norms during clinical examination to avoid making inaccurate diagnostic and treatment plan decisions. At the same time, one needs to acknowledge, as stated by McNamara and Ellis, that “... infinite combinations of dentoskeletal and soft tissue relationships are possible to arrive at a face that is well balanced."

\section{References:}

1. Wahl N. Orthodontics in 3 millenia. Chapter 1: Antiquity to the mid-19th century. Am J OrthodDentofacialOrthop 2005; 127:255-259.

2. Ricketts RM. The biologic significance of the divine proportion and Fibonacci series.Am J Orthod1982;81:351370
3. Peck H, Peck S. A Concept of Facial Esthetics. Angle Orthod1970; 4:284-317.

4. Sushner N. A photographic study of the soft-tissue profile of the Negro population. Am J Orthod 1977; 72(4):373-385.

5. Lines PA, Lines RR, Lines CA. Profilemetrics and facial esthetics. Am J Orthod 1978; 73(6):648-657.

6. Michael E Koury and Bruce N E pker. Maxillofacial esthetics: Anthropometrics of the Maxill ofacial region. J Oral MaxillofacialSurg 1992, 50:806-820.

7. Leslie G Farkas. Anthropometry of the Head and Face. Second edition, New York: Raven Press; 1994.

8. Sutter J $r$, Turley PK. Soft tissue evaluation of contemporary Caucasian and African American female facial profiles. Angle Orthod.1998; 68(6):487-496.

9. Hwang HS, Kim WS, McNamara J A. Ethnic Differences in the Soft Tissue Profile of Korean and European-American Adults with Normal Occlusions and Well-Balanced Faces. Angle Orthod 2002; 72:72-80.

10. Fernandez-Riveiro P, Smyth-Chamosa E, SuarezQuintanilla D, Suarez-Cunqueiro. Angular photogrammetric analysis of the softtissuefacial profile. Eur J Orthod 2003; 25:393-399.

11. Cooke MS, Wei SH. The reproducibility of natural head posture: A methodological study. Am J Orthod Dentofacial Orthop 1988; 93:280-288.

12. Claman L, Patton D, Rashid R. Standardized portrait photography for dental patients. Am J OrthodDentofacialOrthop1990; 98:197-205.

13. Rosemie MA Kiekens, Anne Marie Kuijpers-J agtman, Martin A vant Hof, Bep E vant Hof, Huub Straatman, and J aap C. Maltha. Facial esthetics in adolescents and itsrelationship to "ideal" ratios and angles. Am J OrthodDentofacialOrthop 2008;133:188.

14. William R Prof itt, Henry W Fields J r, David M Sarver .Contemporary Orthodontics, 4th Edition. Mosby Elsevier 2007, pg 176

15. William Arnett G, and Robert T. Bergman. Facial keys to orthodontic diagnosis and treatment planning. Part I. Am J Orthod Dentofacial Orthop 1993; 103:299-312.

16. William Arnett $\mathrm{G}$, and Robert T. Bergman. Facial keys to orthodontic diagnosis and treatmentplanning. Part II. Am J Orthod Dentofacial Orthop 1993; 103:299-312.

17. Sarver DM. Esthetic Orthodontics and Orthognathic Surgery. St.Louis: Mosby; 1998: pg 7.

18. Richard A. Riedel. Aesthetics and its relation to orthognathic therapy. Angle Orthod 1950; 20:168-178.

19. Yuen $\mathrm{S} W \mathrm{H}$, Hiranaka D K. A photographic study of the facial profiles of southern Chinese adolescents. Quintessence International 1989; 20:665-676. 